\title{
Multi-objective Transmission Planning Paper
}

\author{
Xu, Zhao; Dong, Zhao Yang; Wong, Kit Po; Fan, Zhun
}

Published in:

APPEEC09

Link to article, DOI:

10.1109/APPEEC.2009.4918509

Publication date:

2009

Document Version

Publisher's PDF, also known as Version of record

Link back to DTU Orbit

Citation (APA):

Xu, Z., Dong, Z. Y., Wong, K. P., \& Fan, Z. (2009). Multi-objective Transmission Planning Paper. In APPEECO9 (pp. 80821). IEEE. https://doi.org/10.1109/APPEEC.2009.4918509

\section{General rights}

Copyright and moral rights for the publications made accessible in the public portal are retained by the authors and/or other copyright owners and it is a condition of accessing publications that users recognise and abide by the legal requirements associated with these rights.

- Users may download and print one copy of any publication from the public portal for the purpose of private study or research.

- You may not further distribute the material or use it for any profit-making activity or commercial gain

- You may freely distribute the URL identifying the publication in the public portal

If you believe that this document breaches copyright please contact us providing details, and we will remove access to the work immediately and investigate your claim 


\section{Multi-objective Transmission Planning}

\author{
Zhao Xu \\ Centre for Electric Technology \\ Department of Electrical Engineering \\ Technical University of Denmark \\ Kgs. Lyngby, Denmark \\ zx@elektro.dtu.dk
}

\author{
Zhao Yang Dong \\ Department of Electrical Engineering \\ The Hong Kong Polytechnic \\ University \\ Hung Hom, Kowloon, Hong Kong \\ zydong@ieee.org
}

\author{
Kit Po Wong \\ Department of Electrical Engineering \\ The Hong Kong Polytechnic \\ University \\ Hung Hom, Kowloon, Hong Kong \\ eekpwong@polyu.edu.hk
}

\author{
Zhun Fan \\ Section of Engineering Design \\ Department of Management \\ Engineering \\ Technical University of Denmark \\ Kgs. Lyngby, Denmark \\ zf@man.dtu.dk
}

\begin{abstract}
This paper describes a transmission expansion planning method based on multi-objective optimization (MOOP). The method starts with constructing a candidate pool of feasible expansion plans, followed by selection of the best candidates through MOOP, of which multiple objectives are tackled simultaneously, aiming at integrating the market operation and planning as one unified process in the market environment. Subsequently, reliability assessment is performed to evaluate and reinforce the resultant expansion plan from MOOP. The proposed method has been tested with the IEEE 14-bus system and analyses and discussions have been presented.
\end{abstract}

Keywords- Transmission planning, Multi-objective optimization, Electricity market.

\section{INTRODUCTION}

The electricity delivery system, including transmission and distribution, plays an important role in overall electric power system. To fulfill the customer's ever growing demand, constructing new and reinforcing existing transmission networks are of high importance. Properly planned expansion will ensure the healthy operation of the power systems thereby supporting the development of economy and society.

In the past, electricity section was thought naturally a monopoly. Electricity generation, transmission and distribution were vertically-integrated as one system. Expanding transmission network was solely the responsibility of the power system operator, eventually the government. As such, the major concern of transmission planning used to be the investment cost. The transmission planning problem, though dynamic in nature, is often simplified as a static optimization model, minimizing the total investment of network expansion for a single future scenario, subject to a number of constraints [1-3]. In most literature, the static transmission network expansion planning (STNEP) model is typically formulated to minimize the sum of investment cost and the load curtailments caused by lack of transmission capacity, subject to DC or AC load low equation [3-8]. The result is an expansion plan with the least investment with strictly limited load curtailment where necessary.

The least-cost planning model presumes that the power system is vertically integrated, owned, operated and planned by one operator. However, due to the market liberalization, this is no longer the case in many countries, where the integrated power systems have now been split into small, separated and competitive entities. These independent companies provide unbundled services of generation, transmission and distribution, and compete against each other to pursue maximal interests in an open environment. In planning the transmission expansion, their objectives would become rather market oriented, in which both investment and profit prospects would be concerned. On the other hand, the physical topology of transmission network has natural impacts on electricity market operation which should be addressed into the planning process as well. Obviously, the traditional least-cost planning method does not reflect these economic signals and is no longer appropriate in this context.

Recognizing the inherent limitations of the traditional planning approach, the authors previously developed a multiobjective transmission planning model [9]. The developed model integrates the objectives of investment, transmission profit, and market dispatching into one optimization approach, followed by reliability assessment and optimal reinforcement to achieve the final plan. In formulating the candidate expansion plan, practical engineering and management concerns have also been considered. In this work, the model has been further developed to enhance the solution methodology. A superior multi-objective technique i.e. the $\epsilon$ constraint method is applied in this paper due to its capabilities of finding pareto-optimal solutions even with non-convex and discrete objective space [10].

\section{OVERVIEW OF THE PlanNING Method}

Figure 1 presents the structure of the proposed method, where preparation stages for planning study such as demand forecasting and reliability assessment are not included. The method can be divided into three stages, including the initial formulation of the candidate pool for expansion, followed by a multi-objective optimization subject to constraints, and the final security assessment and necessary reinforcement of the expansion plan from MOOP based on the "N-1" criterion.

\section{A. Formulation of candidate pool}

The initial candidate pool for expansion is formulated based on the information of the given system, such as the generation and transmission capacity, the estimated transmission tariffs, the planning scope and corresponding forecasted system demand etc. Human knowledge and empirical rules are applied in this stage to ensure rationality of the candidate line selection with practical engineering and management concerns, which may include but not limited to, network redundancy; environmental factors; financial constraints; estimated construction periods and the planning horizon etc.

The formulation of candidate pool can start from determining the planning horizon and corresponding market 
forecasts. Subsequently, market simulation can be carried to identify the transmission bottlenecks. The pool is then formulated to enhance transmission with redundancy for system reliability. Next, by examining investments and construction periods of individual lines, a number of candidates can be dropped off at this stage to avoid excessive workload in following steps. By taking into account the environmental factors, further reduction of the pool can be made, for example, to avoid a line construction through an important natural reserve.

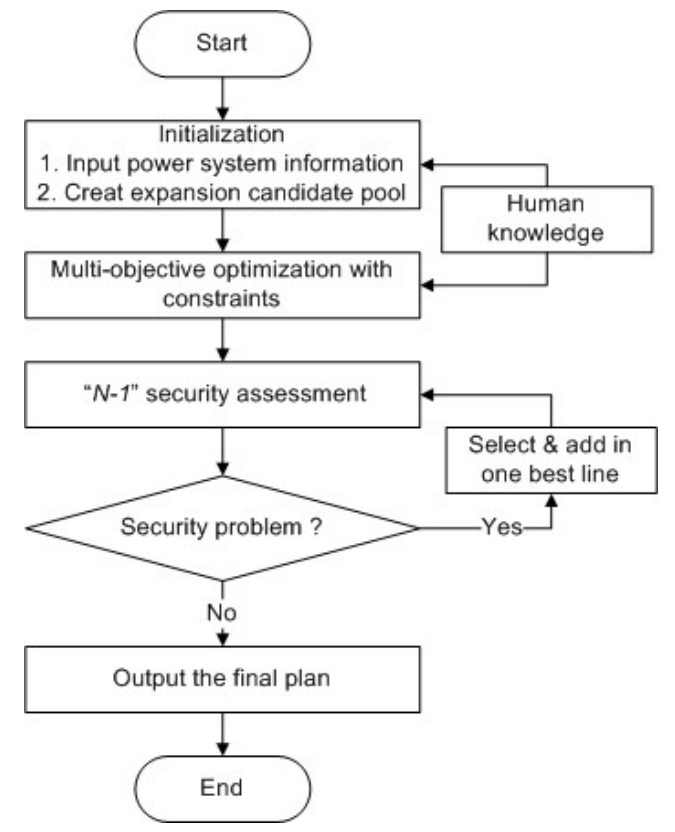

Figure 1. Flowchart of the hybrid planning scheme for TNEP

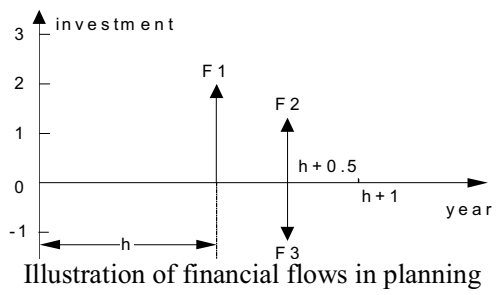

Figure 2. Illustration of financial flows in planning

\section{B. Expansion Plan Selection by MOOP}

This step will select the best expansion plan from the formulated candidate pool by MOOP. To mathematically model the expansion plan, an array is used to represent the candidate lines in each transmission corridor $[3,7,9]$. The proposed planning model include several objectives to maximize the benefit of expansion (BOE), i.e. the transmission service income, while minimizing the overall investment, subject to power flow and security constraints etc. Furthermore, minimization of the generation cost is included since the electricity market should be dispatched at the least generation cost give a System Marginal Price (SMP) auction based market [11]. To account for the time value of money, individual objective is converted to its Net Present Value (NPV), assuming the investment happens at the beginning of the planning year $(h)$ and new circuits are immediately ready to serve [2]. It also assumes the transmission and generation incomes happen at the mid of the planning year $(h+0.5)$. Figure 2 illustrates the financial flow in the planning horizon. The objectives of planning are given in Equations (1-7).

Minimize,

$$
\begin{aligned}
F_{1} & =\boldsymbol{C}^{\boldsymbol{T}} \boldsymbol{\eta} /(1+u)^{h} \\
F_{2} & =f_{2}\left(\boldsymbol{P}_{G}\right) /(1+\mu)^{h+0.5} \\
& =\left[\sum_{i \in N_{G}}\left(a_{i}+b_{i} P_{G i}+c_{i} P_{G i}^{2}\right)\right] /(1+\mu)^{h+0.5}
\end{aligned}
$$

Maximize,

$$
\begin{aligned}
F_{3} & =f_{3}(\boldsymbol{f}, \boldsymbol{\eta}, \boldsymbol{r}, \boldsymbol{d}) /(1+\mu)^{h+0.5} \\
& =\left[\sum_{(i, j) \in \Omega} f_{i j} r_{i j} d_{i j} \frac{\eta_{i j}}{\eta_{i j}+1}\right] /(1+\mu)^{h+0.5}
\end{aligned}
$$

Subject to,

$$
\begin{aligned}
& f_{i j}-\left(x_{i j}^{0}+x_{i j}\right)\left(\delta_{i}-\delta_{j}\right)=0 \\
& \left|f_{i j}\right|-x_{i j} \xi_{i j} \leqslant x_{i j}^{0} \xi_{i j} ; \xi_{i j}=\frac{f_{i j}^{M a x}}{x_{i j}^{0}} \\
& P_{G i}^{M i n} \leqslant P_{G i} \leqslant P_{G i}^{M a x} \\
& x_{i j}=\eta_{i j} r l_{i j} ; 0 \leqslant \eta_{i j} \leqslant \eta_{i j}^{M a x} \forall(i, j) \in \Omega
\end{aligned}
$$

where $F_{1}$ and $F_{2}$ are the total investment of expansion and the generation cost of the system respectively, and $F_{3}$ is the expected profit from the expanded transmission lines, based on the MW-Mile method, in which the transmission cost is computed by multiplying the actual power flow, the distance and the pre-determined unit cost of each transmission line [11]. $\mathrm{C}$ is construction cost vector of new transmission lines; $\eta_{i j}$ is an integer representing the actual new circuit addition in transmission route $i-j$ (bus $i-j$ ) while $\eta_{i j}^{M a x}$ is its upper bound; $\mu$ is the discount rate and $h$ is the planning year; $f_{i j}, d_{i j}$ and $r_{i j}$ are the real power flow, length and pre-determined transmission tariff per unit capacity in \$/MW-Mile respectively for the transmission route $i-j ; r l_{i j}$ is the circuit susceptance of added lines; $x_{i j}{ }^{0}$ and $x_{i j}$ are the initial and total new susceptance in branch $i-j ; P_{G i}$ is real power outputs of generator $I$, and $a_{i}$, $b_{i}$ and $c_{i}$ are the coefficients of the cost function; $\Omega$ is the set of candidate transmission lines for expansion selected at initial stage of planning;

The MW-Mile pricing method allocates the costs based on actual system usage as closely as possible, which is superior to other methods, such as post-stamp and contract path methods [11]. Constraints (4)-(6) are similar to those in DC power flow, while Equation (7) simply updates the circuit susceptance due to expansion. Compared to the model in [9], the use of DC flow constraints effectively reduces the computational time. 
The transmission profit in the planning model can be considered as the difference between transmission customer's payment and service provider's cost in future transactions, which measures the social benefit from the transmission deals. In the marketplace, maximization of social benefits is the first priority and should be reflected into the planning stage. By doing so, market operation and planning can be integrated into one unified process. The investment of expansion is another part of the "costs" which may not be fully recovered by the transmission profit. Thus the planning objective is formulated to maximize the benefit of expansion, namely the profit, while minimizing the planning costs. Moreover, it would be reasonable to assess transmission profit based on realistic market dispatching, and therefore a least cost dispatching objective is included in the planning model. The proposed planning method actually seeks to maximize the overall social benefit from expansion by incorporating various factors of interests in horizon into one optimization approach and tackling multiple objectives simultaneously. It should be noted that the proposed approach is one step further to current practice in transmission planning in countries where competitive market has been established. For example, in Australia National Electricity Market, transmission network service providers (TNSPs) need to meet reliability or market benefit criteria set by the National Electricity Rule in order to be funded for system augmentation. Basically, options analysis to compare the NPV of cost and benefit of different expansion options need to be performed in order to select the most preferred expansion option, and then subject to (mostly) reliability test and/or market benefit test, [15].

\section{Multi-Objective Optimization}

The proposed planning model actually formulates a multiobjective optimization problem with incompatible objectives, subject to a list of constraints. One possible solution is to directly combine all objectives into one by using the weighted sum method [10], which suffers from the difficulty in finding the Pareto-optimal solution, i.e. the genuine optimal solution for all objectives, though with great simplicity. In our previous work, the goal programming method is used in solving the model. In this paper, further development of the planning method has been made to enhance the solution methodology. The planning model is to be solved using a superior MOOP technique, i.e. the $\epsilon$-constraint method, which distinguishes itself by its capabilities of finding pareto-optimal solutions even with non-convex and discrete objective space. The method retains one of the problem objectives, while restricting the rest within user-defined values. In this application, objective $F_{3}$ is retained and the planning model results in a typical nonlinear single-objective optimization problem, which can then be expressed as follows [10]:

$$
\begin{array}{ll}
\text { Maximize } & F_{3}(x) \\
\text { Subject to } & F_{1}(x) \leq \varepsilon_{1} \\
& F_{2}(x) \leq \varepsilon_{2} \\
& G(x)=0 \\
& H_{\text {min }} \leq H(x) \leq H_{\max }
\end{array}
$$

where Eqn. (8) is the same objective defined earlier; and $\boldsymbol{x}$ is the solution vector of the optimization model; objective $F_{l}$ and
$F_{2}$ become soft constraints bounded by pre-defined $\varepsilon_{1}$ and $\varepsilon_{2}$; vector $G$ denotes the equality constraints (load flow equations) defined by Eqn. (10); and vector $H$ is the set of inequalities defined by Eqn. (11) - (12). $\varepsilon$ vectors are user-defined and represent upper bounds of the objectives $F_{2}$ and $F_{3}$, and are usually selected in the range of the minimum and maximum values of the objectives. The solutions obtained from the $\varepsilon$ constraint method are very much dependable on the values chosen for the converted soft constrains. Inappropriate $\varepsilon$ vector may produce inaccurate or erroneous results [10]. The Branch and Bound (BNB) method is used to solve the MOOP model due to its capability of handling mixed integer nonlinear optimization problem, though BNB is limited in dealing with problems of high dimension [12].

\section{Security Assessment}

The expansion plan obtained from the MOOP stage may contain insecure configurations. To ensure the system reliability, the expansion plan is assessed by a list of credible single line outages, i.e. the " $\mathrm{N}-\mathrm{l}$ " criteria, using a base case AC load flow. Should overload happen in any transmission line, best individuals from the rest of the candidate pool are selected to reinforce the network. The security assessment is repeated until no overloading happens in the system and the optimal expansion plan is finalized. Three steps of the security assessment involve contingency screening, N-1 checking, and selection of extra line for reinforcement. Contingency screening is based on the Performance Index (PI) [13]. To avoid "masking effect" [14], the selection is not stopped until no overload happens for two continuous outages [13, 14]. N-1 check is conducted by removing single line according to the contingency list and computing the overload each time. The sum of line overloads in all outages is thus determined. Finally, the best lines to enhance reliability are selected from the rest of the candidate pool based on a cost-benefit analysis, namely the overload relieve per unit line construction cost as defined below,

$$
O c_{l}=\frac{\Delta P_{l}}{C_{l}}
$$

where

$$
\Delta P_{l}=O V L_{B}-O V L_{A}
$$

and $O V L$ is overload in transmission lines (subscripts $\mathrm{B}$ and A stands for before and after); $O c_{l}$ is overload relieve per unit line construction cost. Combinatory effect of adding more than one line is considered in the equation above. Obviously, the lines with larger $O c_{l}$ have the priorities for reinforcement. When $O V L_{B}$, total overload before new line addition, is zero, the expanded system is considered to be " $N-1$ " reliable and the security assessment is terminated without reinforcements.

\section{CASE STUdY}

The IEEE 5-machine, 14-bus system is used in our case study. The total demand for the base year is the $259 \mathrm{MW}$ while the forecasted demand for the planning year $(h=10)$ is increased by 2 times, of which AC optimal power flow proved to diverge, due to overloads happening in transmission lines. Figure 3 presents the demand is unevenly distributed among the buses for the base and planning year. Originally, the system 
has a total of 20 transmission circuits. These circuits have different characteristics such as distance and profit rate of transmission. Since those parameters are not with the original 14 bus system, we have carefully designed the data for our case study, which does not necessarily resemble the actual system.

The planning program is implemented in MATLAB. It starts with selection of the candidate circuits. The vectors in the MOOP model are found by performing minimizations for individual objectives in advance. From the MOOP procedure, the initial expansion plans are generated in Table I, where the candidate routes 3 and 20 are selected. Subsequently, the security assessment (SA) is carried out and two extra lines are added to enhance the reliability, and the resultant investment is increased from 5.24 to $8.75 \mathrm{M} \$$, as a compromise between economy and security.

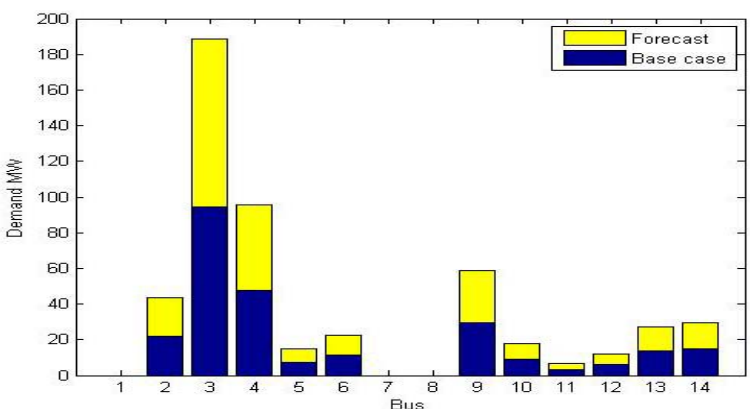

Figure 3. Figure 3 Distribution of demand among buses for base and planning years

\begin{tabular}{cccc} 
TABLE I. & \multicolumn{3}{c}{ SimUlation RESUlts IN CASE STUDY } \\
\hline \hline Route & Max expansion & Before SA & After SA \\
\hline 2 & 1 & 1 & 1 \\
3 & 2 & 0 & 0 \\
5 & 2 & 0 & 0 \\
6 & 2 & 0 & 0 \\
8 & 2 & 0 & 0 \\
10 & 2 & 1 & 1 \\
13 & 1 & 0 & 1 \\
14 & 1 & 0 & 1 \\
18 & 2 & 0 & 1 \\
20 & 2 & 0 & 1 \\
Total investment(M\$) & 5.24 & 8.75 \\
Total capacity added (MW) & 200 & 400 \\
\hline \hline
\end{tabular}

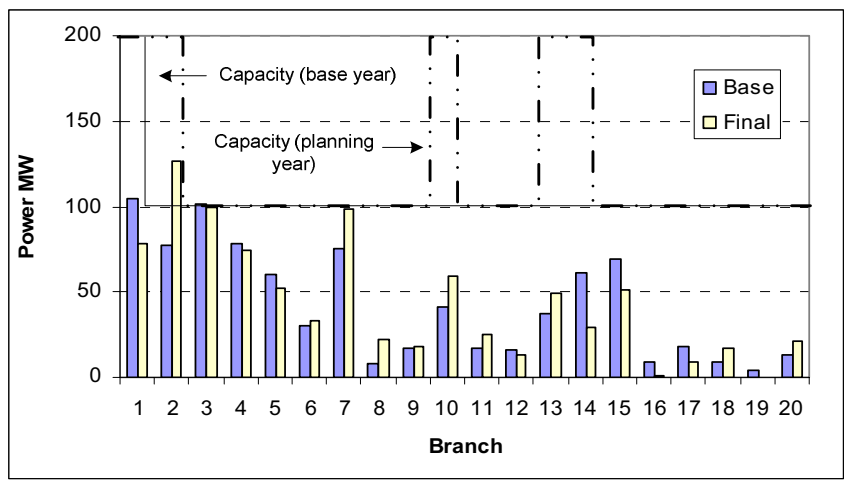

Figure 4. Power flows of transmission routes

To further evaluate the expansion result, optimal power flows have been run for the network before and after expansion, of which the dispatch should generate similar results as a SMP market. Figure 4 compares the resultant real power flows (absolute value) in transmission routes before and after expansion. The capacity limit of each route is also presented for before and after the expansion. It is shown that power flow distribution has been largely changed due to network expansion, which benefits the system with improved security margin of transmission capacity. Particularly, the flow in route 2 has been largely increased over the original limitation after the expansion indicating the necessity of its expansion.

\section{CONCLUSION}

Due to market liberalization, planning power system expansion has to take into account multiple objectives to deal with new concerns and uncertainties that have appeared. Based on our previous work, this paper has further developed the multi-objective planning model by applying a superior $\varepsilon$ constrained MOOP technique and a simplified DC load flow constraints. To resolve the limitation of BNB method and improve computation speed, future research will focus on further simplification of the developed planning model, as well as application of advanced computational intelligence in solving the model.

\section{REFERENCES}

[1] Ahmed H. El-Abiad edited, "Power systems analysis and planning", Proceedings of the Arab School on Science and Technology, Winter Session held in Kuwait, January 21-29, 1981.

[2] X. F. Wang, Fundamentals of Power System Planning, China Electric Power Press, 1993.

[3] H. A. Gil and E. L. da Silva, "A reliable approach for solving the transmission network expansion planning problem using genetic algorithm”, Electric Power System Research 58 45-51, 2001.

[4] M. V. F. Pereira and L. M. V. G. Pinto, "Application of sensitivity analysis of load supplying capacity to interactive transmission expansion planning," IEEE Trans. Power App. Syst., vol. PAS-104, pp. 381-389, Feb. 1985.

[5] R. Romero and A. Monticelli, "A hierarchical decomposition approach for transmission network expansion planning," IEEE Trans. Power Syst., vol. 9, pp. 373-380, Feb. 1994.

[6] R. Romero, R. A. Gallego and A. Monticelli, "Transmission system expansion planning by simulated annealing," IEEE Trans. Power Syst.,vol. 11, pp. 364-369, Feb. 1996.

[7] E. L. Silva, H. A. Gil and J. M. Areiza, "Transmission network expansion planning under an improved genetic algorithm," IEEE Trans. Power Syst., vol. 15, pp. 1168-1175, Aug. 2000.

[8] R. A. Gallego, R. Romero and A. J. Monticelli, "Tabu search algorithm for network synthesis," IEEE Trans. Power Syst., vol. 15, pp. 490-495, May 2000.

[9] Z. Xu, Z. Y. Dong and K. P. Wong, 'A hybrid Planning Method for Transmission Network in a Deregulated Environment', IEEE Transactions on Power Systems, vol: 21, issue: 2, 2006.

[10] K. Deb, Multi-Objective Optimization using Evolutionary Algorithm, John Wiley \& Sons, Ltd, 2001.

[11] K. Bhattacharya, M. H. J. Bollen and J. E. Daalder, Operation of Reconstructed Power System, Kluwer Academic Publishers, 2001.

[12] C.A. Floudas, Nonlinear and Mixed Integer Optimization: Fundamentals and Applications, Oxford University Press, 1995.

[13] T. A. Mikolynnas and B. F. Wollenberg, "An advanced contingency selection algorithm", IEEE Trans. on Power App. and Syst., vol PAS100, No. 21981.

[14] K.F Schafer and J.F Verstege, "Adaptive procedure for masking effect compensation in contingency selection algorithms", IEEE Trans. on Power Systems, vol. 5, iss. 2, May 1990, pp.539- 546.

[15] Australian Energy Market Commission (AEMC), National Electricity Rules, version 21, available on line at: http://www.aemc.gov.au/rules.php 